\title{
La sexualidad en pacientes con cáncer de mama o cérvix sometidas a tratamiento quirúrgico en el Hospital General, Hospital San Vicente de Paúl e Instituto de Cancerología de la Clínica las Américas, Medellín, 1999
}

\author{
María Stella Fernández S. de S.*, Bernarda Ospina de González**, Adela María Múnera Garcés***
}

Recibido: agosto 21/2001 - Revisado: octubre 2/2001 - Aceptado: mayo 30/2002

\section{RESUMEN}

Es necesario tener en cuenta la sexualidad, cuando se va a dar un cuidado de salud integral a la mujer sometida a una mastectomía o histerectomía, a partir del holón erótico y de vinculación afectiva interpersonal, especialmente en la relación de pareja, siendo éste el objeto de esta investigación. Para ella se empleó el método cualitativo de enfoque etnográfico; participaron 27 mujeres, con vida de pareja activa y un año de haber sido sometidas a la cirugía. Se presentó una alteración en el holón erótico del cual surgen cuatro subcategorías: manifestación biológica, mutilación, componente mental y elaboración de la pérdida. Del holón de la vinculación afectiva interpersonal surgen dos subcategorías: satisfacción del deseo del otro y el afecto. Se dieron dos categorías emergentes: el significante de la matriz y el de la menstruación; hay una disminución de la libido y una pérdida de símbolos de feminidad, lo que las llevó a una elaboración de duelo por la pérdida corporal.

Palabras clave: sexualidad, cáncer, mastectomía, histerectomía.

\section{SUMMARY}

When integral health care is going to be given to women who go through a mastectomy or a hysterectomy surgery, it is necessary to keep in mind the sexuality form the erotic holon and from the interpersonal emotional attachment, specially regarding the couple's relationship. These two aspects are the objects of the present research study. An ethnographic approach was used in the study. Twenty-seven women, after a year of having had surgery and with an active sexual life, participated in the study. An alteration in the erotic holon was presented, from which four subcategories emerged: the biologic manifestation, the mutilation, the mental component and the elaboration of the loss. From the holon of the interpersonal emotional attachment, two subcategories emerged: to satisfy other's desire and the affection. Two categories emerged: the significance of the womb and the menstruation. There is a decrease in the libido and a loss of femininity symbols. The corporal loss led the woman to elaborate her grief.

Key words: Sexuality, cancer, mastectomy, hysterectomy.

\section{INTRODUCCIÓN}

Muchas son las inquietudes que despierta el conocimiento y el estudio de la sexualidad humana, más aún cuando el profesional de la salud se interesa por dar un cuidado integral e interdisciplinario como en el caso de la mujer sometida a un tratamiento quirúrgico por cáncer de mama o de cérvix. La comprensión de esta realidad, objeto de este estudio, hace necesario el acercamiento a la mujer para que exprese su sentir y su experiencia en el amplio mundo de su sexualidad a partir del holón erótico y el holón de vinculación afectiva interpersonal, especialmente en la relación de pareja, como lo teoriza Eusebio Rubio (1).

\footnotetext{
* Magíster en Desarrollo Humano, Cinde; profesora titular, Facultad de Enfermería, Universidad de Antioquia.

** Magíster en Orientación y Consejería; profesora titular, Facultad de Enfermería, Universidad de Antioquia.

*** Especialista en Psicología Clínica con énfasis en salud mental, Universidad Pontificia bolivariana; enfermera terapista enterostomal, Medicáncer.
} 
Hasta el momento las investigaciones sobre los efectos de los métodos diagnósticos, del diagnóstico y de los tratamientos quirúrgicos en la esfera de la sexualidad humana son escasas.

Algunos estudios introducen la discusión sobre los efectos psicológicos y físicos de la cirugía de mama y útero, aunque no sean el foco de su investigación. Así, en 1973, Zung reconoce un deterioro sobre la autoestima y la autoimagen en la mujer mastectomizada o histerectomizada, el cual se refleja negativamente en la posibilidad de ejercer una sexualidad normal (2). En 1986, Silberberg y Lubera afirman que... "la histerectomía, la ooforectomía y la mastectomía no impiden por sí mismas la actividad sexual de la mujer"... (3). En 1995, en su estudio en 68 mujeres con cáncer de mama, Ferrero Berlanza y colaboradores encontraron disminuido el bienestar de la mujer con expresión en el área sexual (4), y en 1998, de su estudio en 30 mujeres con cáncer de mama, Arango y colaboradoras reportaron una alteración en la autoimagen y en el autoconcepto, manifiesta en la relación consigo misma y con los demás, expresada en el área sexual, en donde a pesar de existir el deseo, éste se bloquea (5).

El propósito del estudio es comprender la vivencia de la sexualidad en mujeres sometidas a cirugía por cáncer de mama o de cérvix, con el fin de contribuir a una mayor comprensión de su situación y con algunos elementos que permitan un mejor manejo de la misma por parte del equipo profesional de salud.

\section{METODOLOGÍA}

Se realizó un estudio etnográfico. Del diario de cirugía de ginecología y obstetricia fueron seleccionadas las historias clínicas de mujeres sometidas a mastectomía o histerectomía, entre julio de 1997 y julio de 1998, que cumplieran los siguientes criterios de selección: un año de haber sufrido la cirugía por cáncer de mama o de cérvix, tener entre 27 y 58 años de edad, con vida de pareja sexualmente activa, acceder a responder las preguntas con consentimiento informado. Se estudiaron 27 mujeres provenientes del Hospital General de Medellín -cinco por histerectomía y seis por mastectomía; del Hospital San Vicente de Paúl de Medellín -ocho por histerectomía y siete por mastectomía-, y del Instituto de Cancerología de la Clínica Las Américas -una por mastectomía. La investigación contó con el aval de cada una de las instituciones participantes. Las mujeres se entrevistaron hasta cuando la repetición de los contenidos de las respuestas no aportó más información, es decir, se alcanzó la saturación de la información sobre el tema en estudio.

El instrumento utilizado fue la entrevista con preguntas abiertas, elaborada a juicio de las investigadoras sobre la vivencia de la sexualidad posterior al tratamiento quirúrgico. Ésta se realizó en un sitio privado, codificada con un número, grabada y transcrita. Se hizo prueba piloto y las preguntas fueron codificadas a medida que surgieron otras subcategorías. Las entrevistas fueron estandarizadas previamente.

Para el análisis se creó un archivo de datos a partir de la transcripción de las entrevistas. Se codificaron por segmentos que permitieron descubrir conceptos y categorías relacionadas entre sí; se ordenaron y tamizaron esos segmentos, permitiendo descubrir e identificar subcategorías, tendencias y relaciones; se eliminaron aquellas que se salían de lo investigado y se examinaron las evidencias negativas. Para contrastar los datos obtenidos se usó el esquema holístico con base analítica propuesto por Eusebio Rubio en 1996, para estudiar la sexualidad humana.

Finalmente, se organizaron los hallazgos complementados con los testimonios pertinentes y con base en ellos se dan las conclusiones generales y las recomendaciones para el personal de salud.

\section{RESULTADOS}

La vivencia de la sexualidad en las mujeres de este estudio muestra una alteración del holón erótico, que es el fruto de relacionar su cuerpo con el placer sexual. De esta categoría surgen cuatro subcategorías:

1. Manifestación biológica, que corresponde a la expresión verbal consciente de la mujer sobre útero o mama con el concepto implícito de que es una parte erotizada del cuerpo. Tal como lo expresa el sexólogo E. Rubio, sufre una alteración en la referencia que se hace de la apariencia corporal en términos de reconocimiento consciente de esa parte del cuerpo erotizada, expresada por testimonios como: "yo rebajé de peso", "me siento más gordita y apretadita", "me siento mucho mejor que antes, hasta estéticamente".

El cuerpo de la mujer juega un gran papel en el rol genérico que ella desempeña en su vivencia sexual, reforzado por la cultura. Por eso la mujer encuentra una explicación para estas cirugías desde el punto de vista médico, pero no en su pensamiento, donde emergen preguntas, ideas negativas y temores, que dan paso al concepto de mutilación. 
2. La mutilación, expresada verbalmente como la percepción de la pérdida de esa parte erotizada de su cuerpo, es explicable como una castración psicológica que repercute en una deficiencia apreciable de ese cuerpo que por años había considerado íntegro y ahora le da lástima: "[...] siento como un hueco dentro de mí cuerpo"; "yo siento como que me faltara una pieza en el cuerpo".

3. El componente o estructuración mental, dado por la capacidad intelectual que hace posible formar los constructos mentales permitiendo la representatividad y simbolización que la mujer hace de determinadas partes del cuerpo. Aquí el componente mental permite que estas mujeres expresen cómo sus senos eran un atractivo erótico singular y símbolo de feminidad y por tanto de seducción: "sin el seno, uno se siente como un nono", "el seno es el arreglo de la mujer, es lo más importante"; "uno se siente muy incómoda al mirarse al espejo, al vestirse, al no poderse poner las blusitas que usaba antes". Esta simbolización va ligada a lo imaginario femenino, como relacionando lo que es y para qué lo es: "la gente no lo determina a uno para nada".

4. La elaboración de la pérdida afectiva, proceso que mostró cómo la mujer asimila la pérdida del útero o del seno como partes erotizadas que se supone le habían pertenecido, para lo cual sufrió todo un proceso de elaboración de duelo, como lo determina Aguilera (6).

La pérdida de la matriz se ve como una falta de algo que la hace sentir una mujer incompleta, pero al mismo tiempo como liberación para tener relaciones coitales sin temor a otro embarazo.

Estas dos pérdidas, seno y útero, llevan a que la mujer pase por un proceso de elaboración de la pérdida afectiva, que en algunos casos se considera un duelo patológico por lo prolongado en el tiempo, "la tristeza mía fue perder el seno"; "no me voy a poder poner mis blusitas escotadas, voy a tener que vivir como una veterana".

Por eso, esa tristeza converge en su feminidad y toca su holón erótico, y ese erotismo es lo que está en torno al apetito sexual, la excitación sexual el orgasmo y todos los constructos mentales alrededor de esa experiencia, en el estudio se manifestó por la disminución de la libido: "lo que pasa es que sexualmente a mi no me nace; (las relaciones coitales) ya no son satisfactorias para mí. Debido a esta situación, uno como que se va enfriando". Estas expresiones confirman cómo la histerectomía, que no compromete ninguna mutilación visible, puede ser tan importante para la mujer y más si se tiene en cuenta que la matriz carece de terminaciones nerviosas superficiales, de forma que interviene poco en las sensaciones del goce sexual (7).

Para la elaboración del duelo, se dio un mecanismo de defensa de racionalización, acompañado de sentimientos de religiosidad, lo cual las llevó a una resignación ante este evento: "[...] Señor esa es una parte que Tú me la prestaste y ahora Tú la necesitas, te la llevaste. Psicológicamente eso es la obra del cuerpo, como lo creaste. Entonces Tú nos ayudas a que esa parte no nos cree traumas psicológicos de ver que ya... uno, su cuerpo está mutilado". En el estudio realizado en el Instituto Mexicano del Seguro Social (IMSS) en 71 mujeres con histerectomía, 89.6\% utilizaban igualmente este mecanismo de defensa con contenido religioso y $83.3 \%$ se resignaban ante el hecho (8).

Se separa la espontaneidad de esa parte del cuerpo en su relación de pareja y se "acepta" la nueva situación femenina, para dar una solución a la elaboración del duelo: "... si él quiere hablar de amor, de eso, hasta ahí llegamos", "mientras él no me lo recuerde yo estoy bien".

El segundo holón alterado es el de la vinculación afectiva interpersonal que es la capacidad de desarrollar afectos intensos ante la presencia o ausencia, disponibilidad o no de otro ser humano, y tiene su forma ideal en el amor. De allí se originaron las siguientes subcategorías:

1. La satisfacción del deseo del otro que tiene relación con la vivencia que la mujer hace de su sexualidad proyectada en su pareja. Se da una actitud de "resignación", negándose el derecho al disfrute. Como lo expresa la socióloga A. Londoño, la mujer desarrolla una importante vocación de entrega y sacrificio, una sensibilidad extrema por el dolor y el sufrimiento de los demás (5). Esta actitud, en este estudio, se mostró como una resignación que le permite continuar su vida de pareja negándose al derecho del disfrute: "uno ya no siente pues, como esos deseos de estar con ellos; uno está con ellos como porque ellos quiten el deseo... pero no porque a uno le den ganas...", "[...] entonces es lo que yo quiero, como hacerme un tatuaje para que él no me vea eso así... de pronto que uno se decida; no es porque... no... después de la cirugía él no sabe nada, el no me ha visto siquiera las cicatrices, no... él es muy comprensivo".

2. El afecto, tomado en este estudio como la capacidad de la mujer para expresar sus afectos de una manera mesurada y espontánea, permitiendo emerger los sentimientos que, al estar reprimidos, provocan en ella malestar, desplazando la libido 
pero sin apartarse del mundo exterior; como lo expresa Nasio, "jamás nos hallamos tan a merced del sufrimiento como cuando amamos" (6). "El ahora no me busca; llevamos muchos meses sin tener una relación, sin que él esté sobre mí, no tenemos deseos $[\ldots] "$.

$\mathrm{Al}$ analizar los hallazgos de la información se descubren dos categorías emergentes:

1. El significado de la matriz expresada como una parte importante del cuerpo de la mujer y que tiene relación con la identidad femenina. Aunque ésta corrientemente es "invisible", su ausencia la hace visible al sentir cambios corporales en la apariencia de su cuerpo, "se siente uno como más vacío por dentro", "yo así... quedé como un coco y me decían que uno quedaba como un coco... y ya no me importa [...] yo siento como que me quitaron un peso de mi cuerpo". Además, en todas estas pacientes es bien reconocida como una función de la matriz, dar luz, dar nueva vida, "la matriz es importante para tener bebés y nosotros, como yo estoy casada de nuevo, teníamos ganas de tener un hijo..."; la matriz se la deberían sacar a todas las mujeres que no quieran tener hijos...".

Se nota una tendencia a la liberación porque elimina el temor a un embarazo y además se pierde la menstruación.

2. El significante de la menstruación, comprendido como un evento fisiológico exclusivo de la mujer, es expresado como la sangre que sale mensualmente, ayuda en su identidad de género y a aceptar esa condición de mujer, que puede ser de sufrimiento, el cual se percibe como un obstáculo para las relaciones de pareja: "... volví a tener relaciones sin inhibición, porque uno como... con ese período se inhibía"; "no volví a usar toallas, volví a estar limpia a toda hora [...]"; "Uno ya no enferma, ni nada, uno la extraña"; "una señora me dijo que uno envejecía más ligero, porque uno ya no menstruaba y pues... uno como de todos modos va a llegar a la vejez... yo me siento mal... qué se va a hacer, si uno se va a envejecer más ligero [...]".

\section{DISCUSIÓN}

El diagnóstico de cáncer genera dificultades en las relaciones interpersonales, más aún si éste afecta un órgano relacionado con la sexualidad como la mama. En su estudio, Ana Lucía Arango (5) encontró que el $6.6 \%$ de las mujeres tenía cambios sólo a nivel de pareja. "(....) porque aunque tenga el deseo me bloqueo".

La mujer mastectomizada padece un componente psicológico de ansiedad sexual resultado del cambio en su autoimagen e influenciado por su cambio corporal, el cual disminuye su autoconcepto; lo anterior, sumado al duelo por la pérdida corporal, a la promoción de los símbolos de feminidad y de los prototipos de belleza hecha por los medios de comunicación, más a los eventos sociales y a las exigencias de la pareja, son factores que influyen en la sensualidad y llevan a indagar hasta dónde esta situación de duelo no resuelto amerita un acompañamiento más sistemático que el que hasta ahora se lleva, y hasta dónde es un resultado de la intervención médica. Por último, hasta dónde el nivel de afectación del holón de la vinculación afectiva interpersonal en el afecto permitiría afirmar que incide en la capacidad de la mujer de expresar sus afectos.

Además, surge una inquietud sobre lo singular de cada intervención: en la mastectomía la imagen corporal está muy afectada, mientras que en la histerectomía hay mucha melancolía, demostrada por la ausencia intensa del deseo y por la introyección de la libido.

Este estudio pone de relieve la función del otro como semejante y como imagen del espejo en tanto determinantes del malestar de ellas frente a la carencia por pérdida para asegurar su identidad femenina en relación con el goce y el deseo en su relación de pareja.

A pesar de que en la revisión bibliográfica las investigaciones sobre este tema no enfocaron la vivencia de la sexualidad, aparecen inquietudes sobre la alteración en el ejercicio de la sexualidad en las relaciones de pareja y hay un vacío en este tópico en las mujeres histerectomizadas.

\section{CONCLUSIONES}

La sexualidad posterior a la mastectomía o la histerectomía a causa de cáncer de mama o de cérvix uterino está disminuida. Se manifiesta en los testimonios sobre el significado de la mama como un rasgo visible de la identidad como mujer y del útero como un rasgo invisible, que se hace visible ante su ausencia y, por lo tanto, implica una pérdida parcial de aspectos sexológicamente significativos. De esta manera resalta lo simbólico dentro del componente mental, mostrando que las intervenciones quirúrgicas en mama y útero degradan la configuración amable de la autoimagen, perturbando su sexualidad, en tanto que el componente imaginario de la identidad, e identidad de género, necesita estar ligado a determinados rasgos anatómicos que funcionan como semblantes_en este estudio semblantes femeninos_, que de acuerdo con la cultura y la época son resaltados o no. 
De ahí la necesidad de preparar a los profesionales del área de la salud en la atención integral de las mujeres en el pre y el posoperatorio, sobre aspectos que van más allá de lo médico, como la supervivencia, su calidad de vida, sus temores, la mutilación y el reinicio en la vida sexual, con los inconvenientes por sus sentires no expresados y las incomodidades propias de la convalescencia, el posible rechazo de su pareja y las causas del mismo, el de su grupo social o la compasión de otros y la propia por sentimientos de la propia disminución.

Más allá del acto curativo de la medicina, vale la pena remitirse a qué sentimientos experimentan las mujeres sometidas a una mastectomía o histerectomía, la cual representa una lesión y aparece el dolor como un intento desesperado del yo por desprenderse de la conmoción interna aferrándose al símbolo.

Para ellas, un símbolo psicosexual está ligado a otro. Este dolor se dá como una reacción a una pérdida irreversible que para unas es real y para otras imaginaria. Ante esta realidad se asocian sentimientos que se concretan en la reacción ante la amenaza o peligro de perder el objeto amado. En este caso, el amado es el que excita, hace soñar, decepciona, representa el límite, da la imagen, asegura la consistencia de la realidad y hace tolerable la insatisfacción.

Ante estas angustias las pacientes inician procesos de duelo como un intento de alivio, donde el yo desinviste la representación del amado hasta que ésta pierde simbólicamente su vivacidad y deja de ser un cuerpo extraño "fuente de dolor". El duelo es un proceso de desamor. Éste es revivido cada vez que hay una irrupción de amor, en cada acto que recuerde el símbolo _seno o matriz_, como el acto sexual, el mirarse o ser mirada, el vestirse o desvestirse.

Más allá del acto salvador para la vida, estas pacientes se enfrentan a la "muerte", utilizando como único recurso la palabra, la cual el personal de salud con frecuencia ha taponado con el saber y el miedo a enfrentarlas.

\section{RECOMENDACIONES}

La atención pre y posoperatoria en caso de cáncer de mama o de cérvix uterino amerita la intervención de un equipo interdisciplinario que tenga en cuenta, además del trabajo médicoquirúrgico cuidadoso, una serie de intervenciones que procuren el bienestar y la aceptación de las pérdidas no sólo en lo físico sino en lo psíquico, lo social, laboral, doméstico, en fin, en la vida total de la mujer. Por lo anterior, se recomienda:

- Crear un programa de acompañamiento durante la preparación hacia la pérdida y mientras elabora el duelo por la pérdida de esa parte de su cuerpo, para generar un proceso de autoayuda al grupo de pacientes con el fin de socializar sus temores, sus dudas y reforzar sus respuestas positivas a partir de las vivencias personales.

- Reforzar durante la formación universitaria del personal del área de la salud la idea del trabajo interdisciplinario apoyado en criterios de empatía, consenso y autogestión, que ayuden a las mujeres a aceptar la pérdida de una parte erotizada de su cuerpo.

- Realizar una investigación en la que se indague la vivencia del hombre cuya pareja ha sido sometida a mastectomía o histerectomía por cáncer de mama o de útero.

\section{REFERENCIAS BIBLIOGRÁFICAS}

1. Rubio E. Visión panorámica de la sexualidad humana. Rev Latin Sexo 1996; 11 (2): 139-153.

2. Zung W. From art to science: The diagnosis and treatment of depression. Arch Gen. Psychiatry 1973; 29: 328-337. Citado por: Becin Diep R, Ugarta F. Efectos de algunos fármacos e intervenciones quirúrgicas en la vida sexual. En: Antología de la sexualidad humana. México: Porrúa; 1998; 3: 649.

3. Silberberg E, Lubera J. Cancer statistics 1986; 36: 9-25. Citado por: Becín Diep R, Ugarta F. Efectos de algunos fármacos e intervenciones quirúrgicas en la vida sexual. En: Antología de la sexualidad humana. México: Porrúa; 1998; 3: 649.

4. Ferrero BJ, Toledo AM, Barreto MP. Evaluación Conoamericana de Psicología 1995; 27: 87-102.

5. Arango AL, Botero B, Ossa LM. Algunos cambios en la calidad de vida manifestados por varias pacientes con cáncer de mama usuarias de la EPS. Seguro Social Medellín. Universidad Javeriana- Universidad de Antioquia 1998; 40.

6. Aguilera D, Messick J. Control de los conflictos emocionales. México: Editorial Interamericana; 1976; 92-94.

7. Farré JM. Enciclopedia de la sexualidad humana. Editorial Océano. Tomo IV 1993: 9.

8. Valderrama IP, Carbellino S, Domínguez B. Ansiedad y estilos de afrontamiento en mujeres con cáncer cérvicouterino. Rev Latin Psicol 1995; 27(2): 73-86.

9. Londoño A. Hacia una ética del amor propio en las mujeres. En: Memorias Mujer, salud y autocuidado. OPS, OMS 1992; 124-128.

10. Nasio JD. El libro del dolor y del amor. Archipiélago del dolor. Gedisa 1996; 73. 